\title{
Evaluation of Cholesterol and c-Reactive Protein as Prognostic Factors for Survival in Critically Ill Patients
}

\author{
A.G.Elgazzar ${ }^{1}$, M.E.Ibrahim ${ }^{2}$, B.M.Aglan ${ }^{3}$ and D.A.Gerida ${ }^{4}$ \\ ${ }^{1}$ Chest Dept., Faculty of Medicine, Benha Univ., Benha, Egypt \\ ${ }^{2}$ Internal Medicine Dept., Faculty of Medicine, Benha Univ., Benha, Egypt \\ ${ }^{3}$ Cardiothoracic Surgery Dept., Faculty of Medicine, Benha Univ., Benha, Egypt \\ ${ }^{4}$ Critical Care Dept., Faculty of Medicine, Benha Univ., Benha, Egypt \\ E-Mail: D.Gerida@gmail.com
}

\begin{abstract}
The aims of the study were to evaluate serum total cholesterol and serum C-reactive protein as a prognostic factor for survival in critical ill patients. The study population consisted of 50 patients (age range, 18-90 years; median, 55 years; men/women ratio, 2:3) in critical ill patients . Patients' serum levels of total cholesterol and Creactive protein were measured upon admission to citical care unit, three days later, and on the day discharge from ICU. Total cholesterol levels were significantly lower among the non-surviving patients (day 0,115.4 \pm 29.66 $\mathrm{mg} / \mathrm{dL}$; day $3,113.2 \pm 37.19 \mathrm{mg} / \mathrm{dL}$; day of discharge, $104.0 \pm 42.96 \mathrm{mg} / \mathrm{dL}$ ) than surviving patients $($ day $0,142.5 \pm$ $23.93 \mathrm{mg} / \mathrm{dL}[\mathrm{P}=0.001]$; day $3,145.5 \pm 28.17 \mathrm{mg} / \mathrm{dL}[\mathrm{P}=0.001]$; day of discharge $147.5 \pm 35.97 \mathrm{mg} / \mathrm{dL}[\mathrm{P}=$ 0.001]). C-reactive protein levels were significantly higher among the non-survivors (day $0,136.818 .77 \mathrm{mg} / \mathrm{dL} ; \mathrm{day}$ $3,155.1 \pm 18.94 \mathrm{mg} / \mathrm{dL}$; day of discharge $178.5 \pm 22.30 \mathrm{mg} / \mathrm{dL}$ ) than survivors (day $0,68.85 \pm 19.73 \mathrm{mg} / \mathrm{dL}[\mathrm{P}=$ $0.001]$; day $3,63.08 \pm 18.05 \mathrm{mg} / \mathrm{dL}[\mathrm{P}=0.001]$; day of discharge,52.98 $\pm 14.25 \mathrm{mg} / \mathrm{dL} ;[\mathrm{P}=0.001])$. Serum cholesterol and C-reactive protein can be used as markers for predicting survival in critical ill patients. Low cholesterol and high C-reactive protein levels appear as a valuable tool for individual risk assessment in critical ill patient.
\end{abstract}

Keywords: Cholesterol, C-reactive protein, Prognostic factor, Sepsis,Critical ill patient.

\section{Introduction}

The key features of critically ill patient are severe respiratory, cardiovascular or neurological derangement, often in combination, reflected in abnormal physiological observations (e.g sepsis, septic shock, acute kidney injury, adult respiratory distress syndrome, hepatic encephalopathy) [1].

Sepsis is the leading cause of death in patients in the intensive care unit (ICU), and delay in diagnosis retards initiation of appropriate treatment. yet, diagnosis is not always simple, especially in the often- complex critically ill patient in whom the classical signs of sepsis may not be present or may be associated with multiple other pathological conditions and in whom microbiological cultures are frequently negative [2].

Biomarkers, biologic molecules that characterize normal or pathogenic processes and can be objectively measured, have been suggested as a means of aiding diagnosis predicting disease severity and outcome, and monitoring response to therapy. more than 170 such markers have been studied for potential use in septic patients, but perhaps the best known and smember of the pentraxin family of calcium - dependent ligand binding plasma proteins and is synthesized principally by hepatocytes in response to stimulation by cytokines, notably interleukin(IL6) [3].

The plasma half- life of CRP is about 19 hours. In healthy young adults, the normal plasma concentration of CRP is about $0.8 \mathrm{mg} / 1[4,5]$.

CRP is nonspecific prognostic marker that is elevated in other infectious or inflammatory disorders including rheumatoid arthritis, asthma, $\operatorname{COPD}[6,7,8]$ exacerbation systemic lupus, diabetes mellitus, depression, obesity and smoking.Recent evidence suggests that the total cholesterol level can be used as prognostic tool in ICU patients. The increasing trend in total cholesterol level indicates better prognosis of disease outcome. Similarly, the increasing trend in HDL and LDL level also can be used as prognostic tool to determine the survival rate of patient [9].

\section{Methods}

This study was an observational prospective study that was conducted at the Critical Care Department, Faculty of Medicine, Benha university on 50 adult patients suffering from awide range of diseases. They were included in the period from March 2019 to December 2019.

Patients were divided into survivors group which included 30 patients and non- survivors group which included 20 patients.

\subsection{Inclusion criteria}

During a study period of 10 months, 50 adult patients admitted to ICU suffering from the following disease

- Multiple organ failure.

- Heart failure.

- Sepsis, septic shock

- Respiratory failure, pneumonia.

- Encephalopathy.

- Acute Renal failure. 


\subsection{Exclusion criteria}

- Admitted for trauma. Patients with severe trauma had asudden reduction in total serum cholesterol concentration.

- Immune suppressed patient had alteration of the systemic inflammatory response.

- Poisoning

- Patients on statin treatment with change in dosage in the recent six month. Statin use decrease levels of cholesterol and CRP

- Patients newly started with statin drug within last 6 month

- After getting the informed consent from the patient and patient's attenders, history taking regarding age, sex,marital status, chronic medical illness, family history of diabetes mellitus, hypertension are noted down.Blood sample was collected on day of admission ,third day and day of discharge to do lipid profile and CRP. The increase or decrease in both value was compared with outcome of disease .All the patients were examined and the following parameters were evaluated in all cases.

- Total cholesterol, high density lipoprotein, low density lipoprotein, triglycerides and CRP are measured on day of admission (0 hours) ,day3 (72hours)and day of discharge.

- Complete blood count

- Liver function test

- Renal function test

- Arterial blood gas analysis

- Electrolytes

- Random blood sugar

- Chest X Ray

- USG Abdomen

\section{Results}

During the study period of 10 months from March 2019 to December 2019total of 55 ICU patients were recruited in the study.Out of the 50 patients taken up for study, 30 patients got recovered from disease and discharged, while 20 patients died of disease.

\subsection{Age distribution among subgroups}

The 50patients included in the study belong to different age group. Majority were belonging to above 55 years and only 3 patients with the age less than 30yrs were in the study population, of which two survived and one died of disease. Among the 34 patients with the age above 55yrs in the study population, 18 patients survived and 16 patients died.

\subsection{Gender distribution}

The survival rate was less among males $43 \%$ [13] than females $56.7 \%$ [17] while the non survivor rate was less amonge male $45 \%(9)$ than female 55\% [11].

\subsection{Smoking}

The study included 50 patients, 15 patients were smoker $(30.0 \%)$ and 35 patients were no smoker $(70.0 \%)$. There was no statistically significant difference between the survivors and the nonsurvivors (+ve smker/ -ve smoker ratio was $20.0 / 80.0 \%$ vs $45.0 / 55.0 \%$, P-value $=0.059$ ).

\subsection{Diabetes mellitus}

The study included 50 patients, 21 patients had DM (42.0\%) and 29 patients had no DM (58.0\%). There was no statistically significant difference between the survivors and the non-survivors (diabetics/ non- diabetics ratio was $40.0 / 60.0 \%$ vs $45.0 / 55.0 \%$, P-value $=0.726$ ).

\subsection{Hypertension}

The study included 50 patients, 18 patients had HTN (36.0\%) and 32 patients had no HTN (64.0\%). There was no a statistically significant difference between the survivors and the non-survivors (hypertensive/ non- hypertensive ratio was $43.3 / 56.7 \%$ vs $25.0 / 75.0 \%$, P-value $=0.186$ ).

Table (1) Socio demographic and other variables in relation to disease outcome.

\begin{tabular}{lccc}
\hline VVVVV Variable & Sgvvyy Survivors & Non s Non survivors urvivors (22) & P v P value alue \\
\hline Age group & 5 & 3 & 0.132 \\
$<\mathbf{4 0}$ yrs & 0 & 1 & \\
$\mathbf{4 1}$ to 50 yrs & 8 & 5 & \\
$\mathbf{5 1}$ to 60 yrs & 17 & 11 & \\
$>\mathbf{6 0}$ yrs & & & \\
Gender & 13 & 9 & 0.907 \\
Male & 17 & & \\
Female & & 9 & \\
Smoking habit & 6 & 11 & \\
Yes & 24 & & 0.059 \\
No & & 9 & \\
Diabetes mellitus & 12 & 11 & \\
Yes & 18 & & \\
No & & & \\
\hline
\end{tabular}




\begin{tabular}{lccc}
\hline Table (1) Continue & & & \\
\cline { 1 - 3 } Hypertension & & 3 & 0.035 \\
Yes & 13 & 17 & \\
No & 17 & 17 & \\
\hline
\end{tabular}

\subsection{Lipid profile}

In this study, lipid profiles among 50 patients were compared between day-1, day-3 and day of discharge of the study period.In the comparison of total cholesterol among the survivors subgroup on day- 1 and day-3and day of discharge was found to be statistically significant.Similarly, the comparison of HDL, LDL between day-1, day-3and day of discharge among the survivors subgroup was also found to be statistically
significant.Whereas the comparison of total cholesterol, HDL andLDL among the non-survivors sub group was not statistically significant. The comparison of triglyceride , between day-1, day3and day of discharge among the survivors and nonsurvivors subgroups was not statistically significant. It is shown in the Table (2).

Also high CRP was prominent in non survivor group in contrary to low CRP values in survivor group

Table (2) Analysis of lipid profile values among survivors and non survivors on first, third dayand day of discharge.

\begin{tabular}{lccccc}
\hline Survivors (n=30) mean SD & P value & Non Survivors $(\mathbf{n = 2 0})$ mean SD & P value \\
\hline Tc & Day 1 & $142.59 \pm 23.93$ & 0.001 & $115.42 \pm 29.66$ & 0.001 \\
& Day 3 & $145.54 \pm 28.17$ & & $113.22 \pm 37.19$ & \\
HDL & Discharge & $147.56 \pm 35.97$ & & $104.04 \pm 42.76$ & \\
& Day 1 & $37.87 \pm 7.28$ & 0.013 & $33.47 \pm 2.63$ & 0.026 \\
& Day 3 & $39.09 \pm 7.92$ & & $33.29 \pm 2.71$ & \\
LDL & Discharge & $36.58 \pm 4.80$ & & $33.54 \pm 4.22$ & 0.029 \\
& Day 1 & $64.67 \pm 8.45$ & 0.026 & $67.058 \pm 10.04$ & \\
& Day 3 & $69.84 \pm 10.01$ & & $63.76 \pm 7.78$ & 0.007 \\
& Discharge & $69.95 \pm 12.67$ & & $62.38 \pm 9.80$ & \\
& Day 1 & $105.84 \pm 24.95$ & 0.012 & $141.68 \pm 39.15$ & 0.001 \\
& Day 3 & $112.41 \pm 24.13$ & & $134.8 \pm 36.30$ & \\
CRP & Discharge & $118.76 \pm 22.53$ & & $138.5 \pm 26.64$ & \\
& Day 1 & $68.85 \pm 19.73$ & 0.001 & $136.83 \pm 18.77$ & \\
& Day 3 & $63.08 \pm 18.05$ & & $155.14 \pm 18.94$ & \\
& Discharge & $52.98 \pm 14.25$ & & & \\
\hline
\end{tabular}

\subsection{Statistical methods}

Data were statistically described in terms of range, mean $\square$ standard deviation ( $\square \mathrm{SD}$ ), median, frequencies (number of cases) and percentages when appropriate. Comparison of numerical variables between the study groups was done using Mann Whitney $\mathrm{U}$ test for independent samples. P values less than 0.05 was considered statistically significant. For comparing categorical data, Chi square $(\square 2)$ test was performed..Data were then important into (Statistical Package for the Social Science; SPSS version 20.0 ) software for analysis.

\section{Discussion}

Cholesterol levels are adversely affected by infection or organ dysfunction, but the physiologic significance for these decreased concentrations is not known as in Jeyasuriya A et al (2018) study.

C-reactive protein is predominantly produced and secreted by hepatocytes, although other cells including alveolar macrophages may also synthesize CRP [10]. C-reactive protein is thought to represent a measure of cytokine-induced protein synthesis. The relatively short half- life of approximately 19 hours makes it a useful monitor for follow-up of inflammatory response, infection, and antibiotic treatment. In addition, laboratory tests for CRP are easily available and less costly than cytokine tests.

C-reactive protein is a marker of inflammation that has been used to monitor the course of infection and inflammatory diseases [11-13]. Recently, CRP has been seen not only as a biochemical marker of inflammation but also as an active modulator of the inflammatory response. Increasing or persistently high levels, suggesting ongoing inflammatory activity, indicated a poor prognosis, whereas declining values, suggesting a diminishing inflammatory reaction, were associated with a more favorable prognosis. Hence, trends in CRP concentrations during the first 48 hours of ICU admission can be important in helping to decide whether further, and more invasive, diagnostic procedure

Are needed and whether therapeutic interventions 
should be maintained or modified.

In the present study, of 50 patients admitted in the ICU with severe wide range of diseases during their ICU stay we found that hypocholesterolemia and high CRP had a significant prognostic value for predicting mortality as there were lower values for serum cholesterol $(\mathrm{P}=0.001)$ and higher values for serum CRP $(P=0.001)$ in the non-survival group.

There was mean total cholesterol of $115.4 \mathrm{mg} / \mathrm{dl} \pm$ 29.66 in the non-survivor group and $142.5 \mathrm{mg} / \mathrm{dl} \pm$ 23.93 in the survivor group on day of admission achieving a sensitivity of $100 \%$ and a specificity of $100 \%$ and the values continued to decline over the time of ICU stay in the non-survivor group achieving a sensitivity of $100 \%$ and a specificity of $100 \%$ on day of discharge. Also HDL was $33.47 \mathrm{mg} / \mathrm{dl} \pm 2.63$ in the non-survivor group and $37.87 \mathrm{mg} / \mathrm{dl} \pm 7.28(\mathrm{P}=$ 0.013 ) in the survivor group on day of admission while LDL was $64.67 \mathrm{mg} / \mathrm{dl} \pm 8.45$ in the nonsurvivor group and $67.58 \mathrm{mg} / \mathrm{dl} \pm 10.04$ in the survivor group on day of admission

While serum CRP level was mg/L136.83 \pm 18.77 in the non-survivor group and $\mathrm{mg} / \mathrm{L} 68.85 \pm 19.73$ in the survivor group on the day of admission.

A. Jeyasuriya [9] study have considered total cholesterol less than $160 \mathrm{mg} / \mathrm{dL}$ as hypocholesterolemia based on American Heart Association[14] and had found the same result with mean cholesterol value among 33 survivors on day 1 was $120.76( \pm 18.54)$ and day 3 was $145.30( \pm 55.65)$, it is significant. Similarly, the previous study done by Das $\mathrm{S}$ et al among 55 patients in ICU, showed mean cholesterol value among 22 survivors on day 1 was $110( \pm 11)$ and day 3 was $113( \pm 12)$, which was statistically significant [15]. As the total cholesterol value increases during the period of stay in ICU, is proven to be early prognostic indicator of disease outcome. The patients showing increasing trend of total cholesterol levels has increased chances of survival statistically.

Regard HDL, LDL in our study agree with Jeyasuriya et al (2018) study that showed the mean HDL value among 33 survivors on day 1 was $31.70( \pm 3.98)$ and day 3 was $36.39( \pm 11.98)$, it is statistically significant. But in the previous study done by Das S et al among 55 patients in ICU was statistically not significant.

It has been found in Jeyasuriya et al (2018) study that increasing trend in HDL level during the period of hospital stay can also be used as an early prognostic indicator of disease outcome [15].

The mean LDL value among 33 survivors on day 1 was $68.25( \pm 17.14)$ and day 3 was $78.78( \pm 24.74)$ which is statistically significant found in [9]study also deferred from the previous study done by Das S et al showed mean LDL value among 22 survivors on day 1 was $36( \pm 10)$ and day 3 was $42( \pm 8)$, which was statistically not significant. [15] It has been additionally found in [16] study results that increasing trend in LDL level during the period of hospital stay can also be used as an early prognostic indicator of disease outcome. The patients showing increasing trend of HDL levels has increased chances of survival. Further studies are required to confirm these.

F.H. Qian [16] showed similar results with optimum cutoff limit of $>125 \mathrm{mg} / \mathrm{L}$ regard CRP while [17] had found serum CRP (median; IQR) was 32 (20.5-64.5) in the non-survivor group and 10 (6$14)$ in the survivor group $(P>0.001)$ group with optimum cutoff limit of $>14 \mathrm{mg} / \mathrm{dl}$ achieving a sensitivity of $97.6 \%$ and a specificity of $80 \%$ on day of admission.

\section{Conclusion}

Serum cholesterol and C-reactive protein can be used as markers for predicting survival in critical ill patients . Low cholesterol and high C-reactive protein levels appear as a valuable tool for individual risk assessment in critical ill patient.

\section{References}

[1] M. Ostermann , D. Springings The crtically ill patient- Acute Medicinewileyonlinelibrary, Vol. 25(8), , PP.314-325,2017.

[2] C.Pierrakos , J. L.Vincent. Sepsis biomarkers: a review. Crit Care, Vol.14, PP.R15,2010.

[3] D.Thompson, M.B. Pepys, S.P.Wood The physiological structure of human C-reactive protein and its complex with phosphocholine. Structure, Vol.7, PP.169-77,1999.

[4] B. Shine, F.C. De Beer, M.B.Pepys Solid phase radio immunoassays for human C-reactive protein. Clin Chim Acta,Vol.117, PP.13-23,1981.

[5] M. Raitakari, K. Mansikkaniemi, J. Marniemi, Distribution and determinants of serum highsensitive C-reactive protein in a population of young adults: the cardiovascular risk in young Finns study. J Intern Med, Vol. 258, PP.428$34,2005$.

[6] F.H. Qian, Q. Zhang, L.F. Zhou, High-sensitivity C-reactive protein: a predicative marker in severe asthma. Respirology, Vol. 13, PP.664-9,2008.

[7] M. Dahl, Genetic and biochemical markers of obstructive lung disease in the general population. Clin Respir J,Vol.3, , PP.1212009

[8] R. Kalhan, B.T. Tran, L.A. Colangelo, Systemic inflammation in young adults is associated with abnormal lung function in middle age One, Vol.5, PP.e11431,2010.

[9] A. Jeyasuriya, A.K. Badrinath, S. Nagalingam , Cholesterol levels the prognostic significance in ICU patients. Int J Adv Med, Vol.28, PP.412425,2010.

[10] P.C. Hebert, G. Wells, M.A. Blajchman, A multicenter, randomized, controlled clinical trial of transfusion requirements in critical care. $\mathrm{N}$ Engl J Med, Vol. 340(6), PP.409-417,1999.

[11] D. Cook, G. Guyatt, R. Hall, A comparison of sucralfate and ranitidine for the prevention of 
upper gastrointestinal bleeding in patients requiring mechanical ventilation. Canadian Critical Care Trials Group. N Engl J Med,Vol. 338(12), PP. 791-7,1998.

[12]

D.J. Cook, B.K. Reeve, G.H. Guyatt, Stress ulcer prophylaxis in critically ill patients. Resolving discordant meta-analyses. JAMA,Vol. 275(4), PP. 308-14,1996.

[13]

M.S.Cooper, B.M. Stewart, Adrenal insufficiency in critical illness. J Intensive Care Med, Vol. 22(6), PP. 348-62,2007.
[14] M.H. Criqui , Very low cholesterol and cholesterol loweing. American Heart Association , Vol.71, , PP. 59,1994.

[15] S. Das, S. Bhargava, A. Manocha, The prognostic value of hypocholesterolemia in sepsis. Asian J pharm Biol Res, Vol.1 (1),, PP.416,2011.

[16] P. Ortega-Deballon , F. Radias, O. Facy, Creactive protein is an early predictor of septic complications after elective colorectal surgery. World J Surg,Vol. 34(4), PP. 808-14,2010.

[17] D. Memis, O. Gursoy, M. Tasdogan, High C-reactive protein and low cholesterol levels are prognostic markers of survival in severe sepsis. J Clin Anesth, Vol. 19(3), PP.186-91,2007. 